\title{
A Case of an Tubular Foreign Body in the Paranasal Sinus After Penetrating Orbital Injury
}

\author{
Sang Woo Kimª, Kyoung Rai Cho ${ }^{\mathrm{a}, \mathrm{b}}$
}

\begin{abstract}
We report a case of a 13-year-old male patient who was referred from the emergency room with a retained tubular foreign body in the right orbit extending into the right sphenoid sinus. The foreign body was removed through endoscopic exploration without leaving an external scar.
\end{abstract}

Keywords: Paranasal sinus; Foreign body

\section{Introduction}

A foreign body in the paranasal sinus is very rare, particularly in the frontal and ethmoid sinuses in comparison to the maxillary and sphenoid sinuses [1]. Maxillofacial foreign bodies are mainly traumatic in origin, and they occur directly as a result of external trauma to the respective sinus or indirectly through orbital or palatal injuries [2]. Foreign bodies may cause ophthalmic complications including diplopia, eyeball movement disorders, visual disturbance, cellulitis and abscess. These conditions may also result in rhinologic complications such as nasal obstruction, mucopurulent rhinorrhea, hyposmia and cerebrospinal fluid rhinorrhea [3]. Foreign bodies detected in the orbit, anterior cranial fossa, or the paranasal sinus may consist of different kinds of substances such as pencils, wood, plastic, glass, metal and bullets [3-6]. The management of these cases depends on the object's size, shape, composition and location, as well as the degree and location of the external injury [7].

The authors experienced a case of a foreign body detected in the orbit and the paranasal sinus which was caused by penetration of the periorbital area. Also, the removal of the foreign body was performed by endoscopic surgery without an external incision; therefore, we report this case with a bibliography.

\section{Case Report}

A 13-year-old male patient came to the emergency room (ER) with a $2 \mathrm{~cm}$ linear eyelid laceration after a fall in the classroom (Fig. 1). At that time, he had several minutes of syncope, but he had no relevant past medical history. Also, he did not have any noticeable nasal, ocular or facial symptoms. Physical examination of both nasal cavities and the eyes was normal except for eyelid laceration, so he received primary repair of the laceration in the ER. Then, he underwent paranasal CT, and an abnormal finding was detected in his CT scan. There was a non-enhancing, air-filled tubular structure in the right orbit extending into the sphenoid sinus (Fig. 2, 3).

The patient underwent endoscopic foreign body removal under general anesthesia; ethmoidectomy and sphenoidectomy were performed through an endoscopic approach without

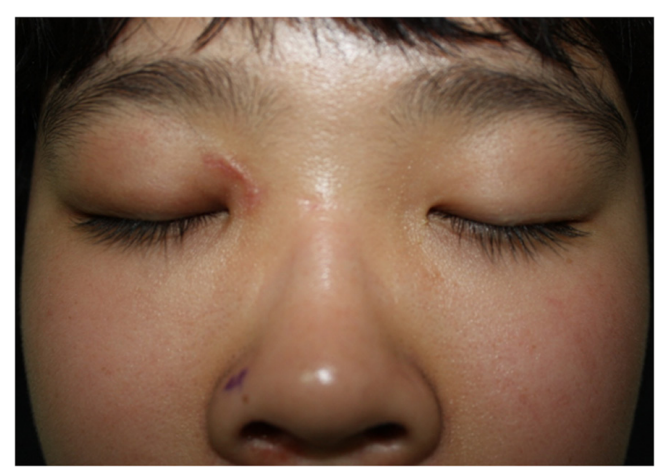

Figure 1. Right medical canthus scar tissue (about a 2 $\mathrm{cm}$ sized laceration). 


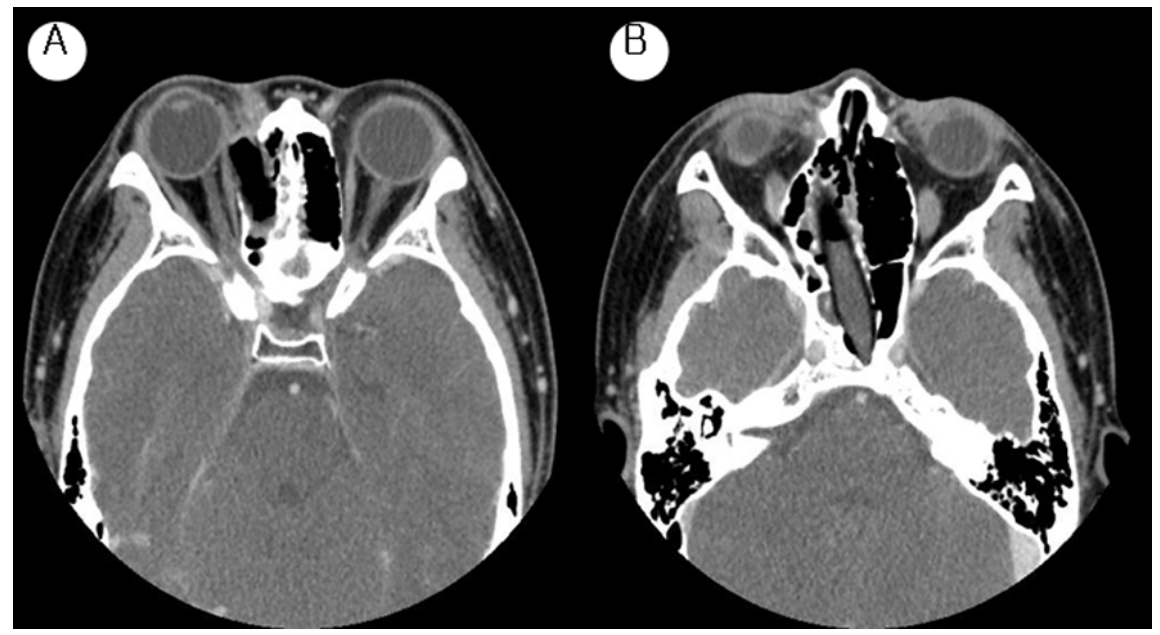

Figure 2. Paranasal sinus CT axial view. There is an air-filled non-enhancing tubular structure extending from the right orbit to the posterior wall of the sphenoid sinus.

an external incision; the middle part of the foreign body was initially incised and then the rest of the body was partially removed. We found that the removed foreign object was an $8 \mathrm{~cm} \times 1 \mathrm{~cm}$ ballpoint pen (Fig. 4). After the surgery, the patient has had no complications up to the present and he currently attends follow-up visits (Fig. 5).

\section{Discussion}

When we assess a patient who is suspected to have a foreign body in the orbit and the nasal cavity, it is important to carefully evaluate his or her underlying symptoms, the history and physical examination. However, if the patient loses con- sciousness due to the trauma or has an asymptomatic foreign body in the cavities, the exact diagnosis can be delayed [ 1 , 8].

A CT scan, which demonstrates a foreign body's location, size, shape and composition, is considered as the most beneficial imaging study [9]. Especially in the case of an injury caused by penetration of the orbit, an imaging study is essential for determining the presence of an intraorbital foreign body and its location. However, identifying the exact location of nonmetallic objects such as those made of wood, plastic or glass on a CT scan is problematic. Therefore, an MRI which gives clear pictures of the soft tissue structures would be a better imaging tool. Therefore, if a foreign body fails to be diagnosed using an X-ray in a patient suspected to

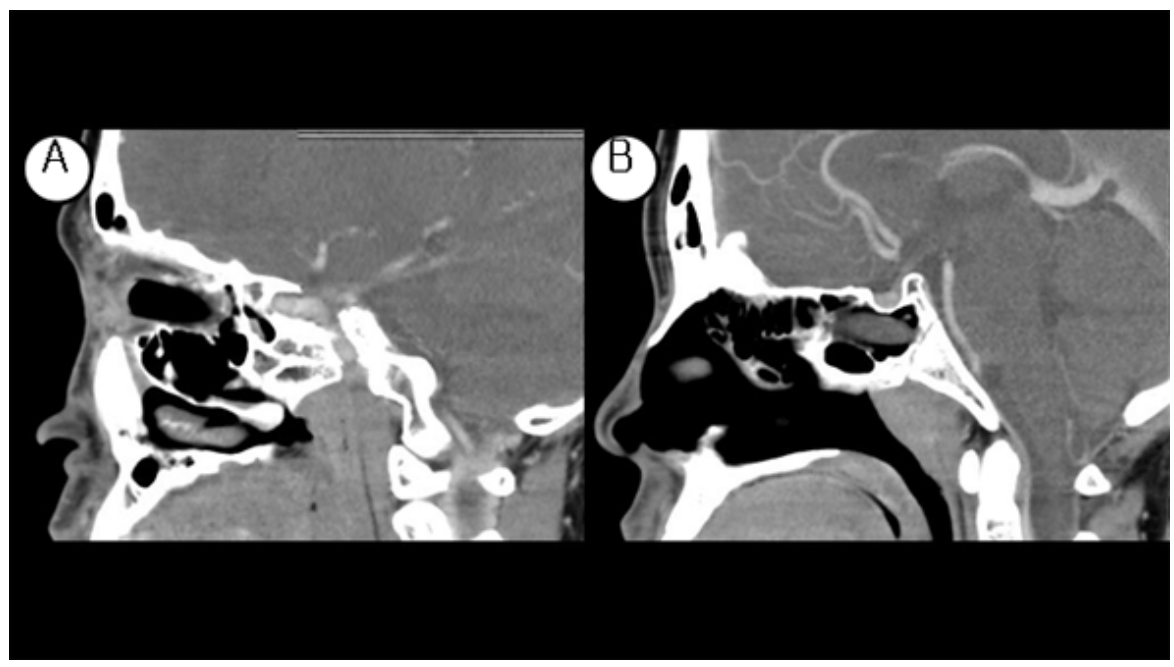

Figure 3. Paranasal sinus CT (sagittal view). There is an air-filled non-enhancing tubular structure which is located in the right orbit and extends to the posterior wall of the sphenoid sinus. 


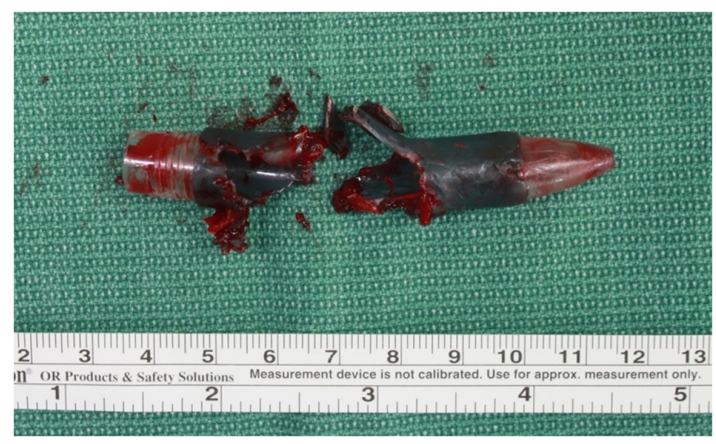

Figure 4. Removed foreign body $(8 \times 1 \mathrm{~cm}$ sized ball pen).

have a foreign body and who has a facial injury, MRI would be a useful diagnostic tool for this patient [9]. In this case, even though the object, which was suspected to be a foreign body, did not appear on a contrast-enhancing CT scan, we were able to diagnose the foreign body in the orbit and nasal cavities by visualizing an air-filled tubular structure on the CT scan.

In order to give appropriate surgical treatment for a patient with a foreign body, clinicians need to obtain detailed medical information on the foreign body, including its location, size, shape and composition, prior to the operation [10]. Moreover, clinicians need to conduct minimal invasive surgical procedures when removing a foreign body in the orbit, orbital tissue, or nasal cavities. In the case of a patient who has a laceration or fistula, clinicians usually remove any foreign body present through the wound site. In the case of a patient whose laceration has healed, clinicians can remove the foreign body by approaching the region of the laceration [11]. In this case, we attempted to remove the patient's foreign body by using an endoscopic approach, and not by approaching the region of the laceration because the foreign body was positioned over the ethmoid sinus and the sphenoid sinus. In addition, foreign body removal was safely performed in the patient without any specific complications. In the case of a patient who has ocular symptoms due to penetration of the orbit or who has an accompanying intracranial injury, he or she should undergo surgical removal under the collaboration of specialists from other clinical departments, such as ophthalmologists and neurosurgeons.

\section{Conflict of Interest}

None.

\section{Disclosure}

All authors significantly contributed to and agreed upon the content of the manuscript and also there is no financial and material support or funding.

\section{References}

1. Sharma R, Minhas R, Mohindroo N. An unusual foreign body in the paranasal sinuses. Indian J Otolaryngol Head Neck Surg. 2008;60(1):88-90.

2. Payne RF. FB in frontal sinus. Br J Radiol. 1967;40:778780 .

3. Kitajiri S, Tabuchi K, Hiraumi H. Transnasal bamboo foreign body lodged in the sphenoid sinus. Auris Nasus Larynx. 2001;28(4):365-367.

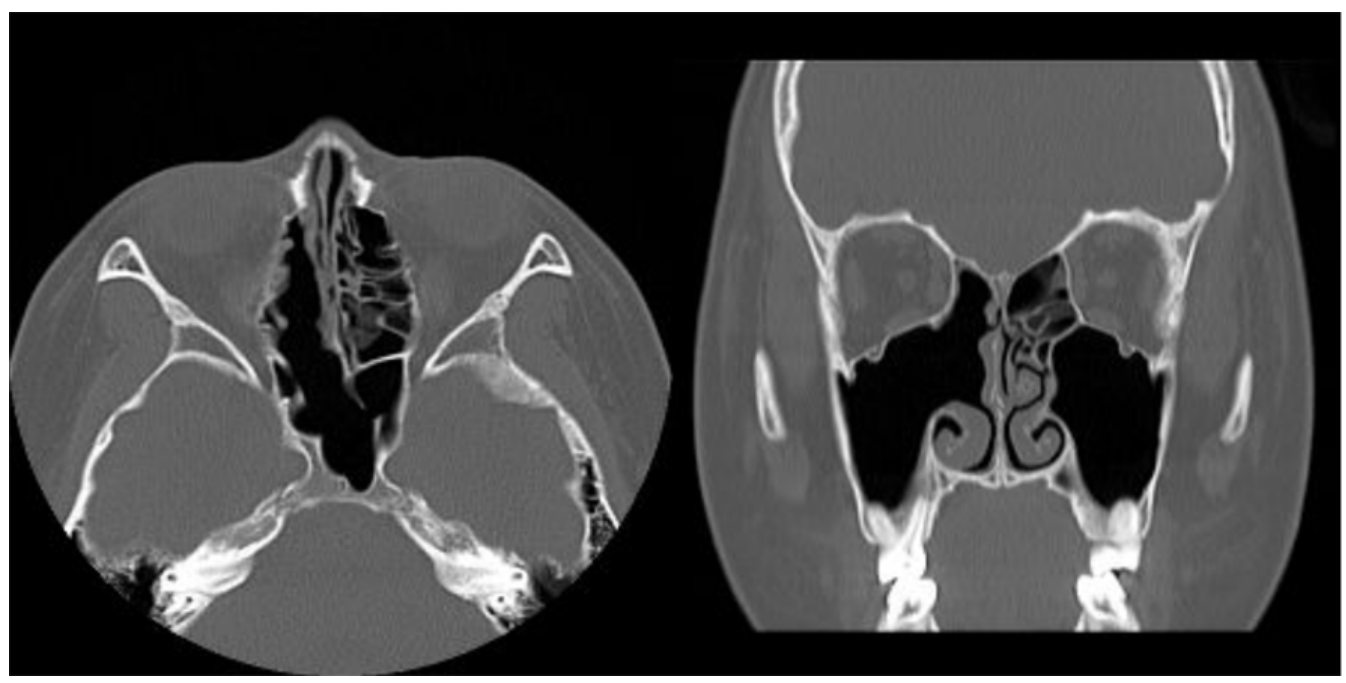

Figure 5. Postoperative paranasal CT findings (axial and coronal view). 
4. Tay AB. Long-standing intranasal foreign body: an incidental finding on dental radiograph: a case report and literature review. Oral Surg Oral Med Oral Pathol Oral Radiol Endod. 2000;90(4):546-549.

5. Dimitrakopoulos I, Lazaridis N, Karakasis D. Unusual retained foreign body in the orbit. J Oral Maxillofac Surg. 1991;49(4):420-421.

6. Davis GA, Holmes AD, Klug GL. Delayed presentation of transorbital intracranial pen. J Clin Neurosci. 2000;7(6):545-548.

7. Tsao YH, Kao CH, Wang HW, Chin SC, Moe KS. Transorbital penetrating injury of paranasal sinuses and anterior skull base by a plastic chair glide: management options of a foreign body in multiple anatomic compart- ments. Otolaryngol Head Neck Surg. 2006;134(1):177179 .

8. Kim HK, Chung WS. Clinical experience of intraorbital foreign body. J Korean Ophthalmol Soc. 1997;38:177184.

9. Weisman RA, Savino PJ, Schut L, Schatz NJ. Computed tomography in penetrating wounds of the orbit with retained foreign bodies. Arch Otolaryngol. 1983;109(4):265-268.

10. Simha A, John M, Albert RR, Kuriakose T. Orbito-sinal foreign body. Indian J Ophthalmol. 2010;58(6):530-532.

11. Lim JH, Kwak MS. A case report of retained intraorbital metallic foreign body removal. J Korean Ophthalmol Soc. 1999;40:1127-1132. 